\title{
La relación entre el gobierno corporativo y la valoración, apalancamiento y desempeño financiero en Colombia
}

\author{
MENESES CeRón, LUIS ÁNGEL \\ Universidad Cooperativa de Colombia \\ Correo electrónico: luis.menesesc@campusucc.edu.co \\ Carabalí Mosquera, JAIME ANDRÉS \\ Universidad ICESI (Colombia) \\ Correo electrónico: jacarabali@icesi.edu.co \\ PÉREZ PACHECo, CAMILO ANDRÉS \\ Universidad Nacional Abierta y a Distancia (Colombia) \\ Correo electrónico: camiloa.perez@unad.edu.co
}

\begin{abstract}
RESUMEN
La literatura financiera y la evidencia empírica en los mercados globales señalan que aquellas compañías que gestionan adecuadamente sus grupos de interés fortalecen y divulgan sus políticas de gobierno corporativo, alcanzan mayor efectividad en su funcionamiento institucional. En el contexto de un mercado emergente, este artículo estudia la relación de la calidad de las prácticas de gobierno corporativo con la valoración, apalancamiento y desempeño financiero de empresas colombianas durante el periodo 2008-2018. A partir de la teoría de la dependencia de recursos y de agencia se establecen predicciones sobre las relaciones entre las variables de interés. Se examinaron empresas que cotizan en la Bolsa de Valores de Colombia y se empleó un índice de Bloomberg para medir la calidad de las prácticas de gobierno corporativo. Se utilizaron modelos de regresión en un panel de datos no balanceado incluyendo efectos fijos de tiempo y empresa. Los resultados indican que existe una relación positiva entre el índice de gobierno corporativo y las medidas de valoración y desempeño financiero de las empresas, y negativa frente a la medida de apalancamiento total. Estos resultados revelan que las buenas prácticas de gobierno corporativo conllevan un mejor funcionamiento al interior de las empresas, lo que se refleja en una mejor performance en el mercado a través del tiempo.
\end{abstract}

Palabras clave: gobierno corporativo, valoración, apalancamiento financiero, desempeño corporativo, COLCAP.

Clasificación JEL: G10; GQ5; G30; G34.

MSC2010: 97M30.

Artículo recibido el 6 de enero de 2020 y aceptado el 25 de febrero de 2021. 


\title{
The relationship between corporate governance, valuation, leverage and financial performance in Colombia
}

\begin{abstract}
Financial literature and empirical evidence in global markets indicate that those companies that adequately manage their stakeholders, strengthen and disclose their corporate governance policies, achieve greater effectiveness in their institutional operation. In the context of an emerging market, this article studies the relationship between the quality of corporate governance practices and the valuation, leverage and financial performance of Colombian companies during the period 2008-2018. Based on the theory of resource and agency dependency, predictions are established about the relationships between the variables of interest. Companies listed on the Colombian Stock Exchange were examined and a Bloomberg index was used to measure the quality of corporate governance practices. Regression models were used on an unbalanced data panel including time and company fixed effects. The results indicate that there is a positive relationship between the corporate governance index and the financial performance and valuation measures of companies, and a negative relationship with the total leverage measure. These results reveal that good corporate governance practices lead to better functioning within companies, which is reflected in a better performance in the market over time.
\end{abstract}

Keywords: corporate governance, valuation, financial leverage, corporate performance, COLCAP.

JEL classification: G10; GQ5; G30; G34.

MSC2010: 97M30.

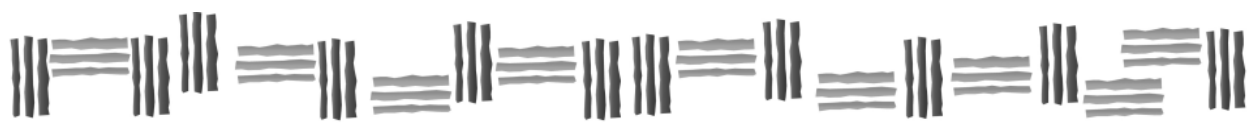




\section{Introducción.}

La evidencia empírica en los mercados competitivos globales sugiere que, aquellas compañías que gestionan adecuadamente sus grupos de interés fortalecen y divulgan sus políticas de gobernanza, logran una mayor efectividad institucional. En este sentido, el estudio del gobierno corporativo y su efecto en el desempeño de la empresa, en mercados desarrollados y emergentes, se ha convertido en una preocupación recurrente para los académicos e investigadores en el campo de la gestión y las finanzas corporativas.

El gobierno corporativo se concibe como un sistema de gestión, que considera optimizar las prácticas directivas en las organizaciones, esperando mejorar la confianza en los grupos de interés, y así, los resultados a futuro (Méndez \& Rivera, 2015). Con base en esta noción, Castro y Cano (2004) señalan que el objetivo central de la teoría del gobierno corporativo es incrementar la confianza de los interesados y eliminar las gestiones fraudulentas de las organizaciones. Esto es posible gracias a los nuevos modelos de gestión corporativos y a los efectos de la divulgación de la información, ya que, al ser un principio fundamental, hace que estos grupos de interés realicen seguimientos y control en el desempeño de las organizaciones (Acero \& Alcalde, 2012).

Las prácticas de gobierno corporativo aportan una solución a la crisis de confianza que se percibe en las diferentes organizaciones, tanto a nivel público como privado. El objetivo de éstas es asegurar la buena administración de los recursos para proteger los derechos de los grupos de interés. Un grupo de interés (stakeholder como se conoce en inglés) lo componen las personas, organizaciones o empresas que tiene interés en la compañía. Los grupos de interés lo componen los trabajadores, accionistas, clientes, proveedores, organizaciones gubernamentales que se encuentren vinculadas, $u$ otro tipo de colectivos civiles. Para ello, se genera una dinámica interna que analiza la estructura organizacional, las políticas y división de funciones, las directrices estratégicas, la administración de riesgos, el manejo de información, reportes y la infraestructura tecnológica para que las políticas de gestión sean más influyentes tanto en la organización como en el entorno (Méndez \& Rivera, 2015).

En los últimos años, la literatura gerencial ha centrado su atención en las prácticas de gobierno corporativo, considerándolo un elemento fundamental de los estudios de la teoría de la empresa. Esto se debe a que su dinámica provee a los directivos y accionistas de mecanismos que permiten diseñar normas encaminadas a la minimización de conflictos de interés, y la consecuente mejora y transparencia en la toma de decisiones, lo que se traduce en una mejora del desempeño empresarial a largo plazo (Escobar et al., 2016). En esta misma línea, Paz (2004) sostiene que las reglas de buen gobierno pueden ser una pieza fundamental para incrementar el valor bursátil de las empresas y, por consiguiente, reducir sus costes de capital y ensanchar en términos de tamaño, profundidad y liquidez las bases del mercado de capitales. La experiencia internacional en la materia permite predecir, además, que la velocidad de implantación del buen gobierno es directamente proporcional a la intensidad de la convicción de los emisores acerca de su rentabilidad. Por otra parte, la Organización para la Cooperación y el Desarrollo Económico (OECD, 2004) resalta que el buen gobierno corporativo es un elemento crucial en la búsqueda del crecimiento económico en regiones con economías emergentes como Latinoamérica. Las economías emergentes dependen del sector privado como un vehículo eficiente para la creación de bienestar, que está subordinado al incremento de las inversiones, la eficiencia del mercado de capitales y el desempeño de las empresas.

Ahora, si bien es claro que existen múltiples investigaciones sobre gobernanza a nivel de mercados desarrollados, dichas relaciones en el contexto propio de los mercados emergentes no se han investigado lo suficiente. En estas economías la dinámica empresarial, vigilancia institucional de los mercados y su tamaño, hacen que el patrón de regulación o autorregulación sea muy heterogéneo. En Latinoamérica, Brasil es el mercado que evidencia los mayores avances, dado que posee instituciones enfocadas en estimular y canalizar el interés por formar mejores criterios de buen gobierno corporativo. Otra experiencia relevante es el caso de México, que ha desarrollado numerosos códigos corporativos. Además, ha establecido una amplia legislación para empresas estatales y privadas que cotizan o no en la Bolsa Mexicana de Valores. En Colombia, la discusión sobre la gobernanza revela 
un notorio desarrollo en las últimas dos décadas gracias a tres hechos sustanciales: la expedición de la Resolución 275 de mayo de 2001, la Ley 964 de 2005 y el establecimiento del Proyecto Código País. Estas legislaciones han recibido el apoyo del Gobierno Colombiano, por intermediación del Ministerio de Hacienda y Crédito Público y la Superintendencia Financiera, articulado con la Bolsa de Valores de Colombia y otras entidades privadas (Oneto \& Nuñez, 2015).

Bajo este contexto, el presente estudio busca ahondar en esta dinámica organizacional y determinar la relación entre el índice de gobernanza y el desempeño de la empresa en un mercado emergente. Para ello, se realiza un análisis con datos de empresas que cotizan en la Bolsa de Valores de Colombia y hacen parte del índice bursátil COLCAP. Para medir la calidad de la gobernanza se utiliza un índice de gobierno corporativo diseñado y calculado por Bloomberg. Para establecer las relaciones teóricas que existen entre estas variables utilizamos los marcos definidos por tres corrientes dominantes: la teoría de la agencia (Jensen \& Meckling, 1976), la teoría de la dependencia de los recursos (Pfeffer \& Salancik, 2003) y la teoría del gobierno corporativo bajo condiciones de incertidumbre (Holmstrom, 1979). La combinación de estas perspectivas permite configurar una visión holística y crítica de la relación entre la estructura de gobierno corporativo y el desempeño de la empresa en el ámbito del mercado colombiano.

A nuestro conocimiento, este documento es el primer estudio que hace uso de índices corporativos de Bloomberg que miden gobernanza, con una estructura de análisis de panel de datos no balanceados, para la estimación de la relación gobierno-desempeño, aplicado al caso de Colombia. Este artículo se divide en cinco secciones. La segunda sección presenta la revisión de la literatura y establece las relaciones teóricas que se predicen de las tres perspectivas que utilizamos. La tercera sección contiene los aspectos metodológicos y la estrategia econométrica. Posteriormente, en la cuarta se presentan los resultados y en la quinta se culmina con las conclusiones del estudio.

\section{Revisión de literatura.}

En los últimos años, la literatura financiera ha centrado su atención en las prácticas de gobierno corporativo, considerándolo un elemento fundamental de los estudios de la teoría de la empresa. Las buenas prácticas de gobierno corporativo proveen a los directivos y accionistas de mecanismos que permiten diseñar y establecer normas encaminadas a la minimización de conflictos de interés y la consecuente mejora y transparencia en la toma de decisiones. Estos son factores que se traducen en un mejoramiento del desempeño empresarial y que se sustentan en la teoría de la agencia (Escobar et al., 2016). Con relación a la dependencia de recursos, debido a su escasez y ubicuidad, los recursos que una empresa necesita no pueden ser completamente producidos internamente. Eso implica que, necesariamente, las empresas deben establecer relaciones con otras para operar. Dado que la distribución de la propiedad de los recursos es asimétrica, las empresas establecen relaciones contractuales con un conjunto preferencial de empresas. Estas relaciones contractuales pueden fomentar un determinado tipo de gobernanza corporativa y, consecuentemente, cambios en el desempeño de la empresa. Ahora, si bien es claro que existen múltiples investigaciones sobre gobernanza a nivel de mercados desarrollados, es necesario que, a través de la academia, se continúe explorando dichas relaciones en el contexto propio de un mercado emergente como el colombiano. En esta sección se describe la forma en la que los trabajos más relevantes han abordado esta cuestión. Destacamos una amplia variedad de trabajos aplicados a diferentes regiones del mundo.

Brown y Caylor (2004) diseñaron una medida gobierno corporativo, Gov-Score, compuesta de 51 factores que abarcan ocho categorías de gobierno corporativo: auditoría, junta de directores, estatutos, educación de directores, compensación de ejecutivos y directores, propiedad, prácticas progresivas y estado de incorporación. Calcularon la medida de gobernanza para 2327 empresas individuales, utilizando datos obtenidos del Institutional Shareholder Services (ISS) para Estados Unidos. Posteriormente, relacionaron Gov-Score con el desempeño operativo, la valoración y el pago a los accionistas, encontrando que las empresas mejor gobernadas son relativamente más rentables, más valiosas y pagan más dividendos a sus accionistas. 
Haynes y Hillman (2010) sostienen que el valor de los recursos gerenciales reside en el capital humano y social de todos los miembros del equipo gerencial. Por una parte, el capital humano consta de elementos como la experiencia, el conocimiento, la reputación y las habilidades de los miembros del equipo directivo, mientras que, por otra, el capital social se refiere a la red relevante que podría utilizarse en beneficio de una empresa en aras de aprovechar oportunidades en diferentes contextos. Por consiguiente, este estudio examinó el efecto de amplitud y profundidad del capital de la junta sobre las decisiones de cambio estratégico, a saber, variación y desviación estratégicas. En esa línea de análisis, la variación estratégica estuvo soportada sobre seis ratios de asignación de recursos de alta incidencia sobre el desempeño empresarial. A la postre, su hallazgo central coincide, al señalar que el capital de la junta es un recurso útil para empresas, con la amplitud de conocimientos, experiencias y lazos sociales que brindan la oportunidad a las empresas para desviarse de las estrategias anteriores y de normas estratégicas de la industria.

Posteriormente, Iqbal et al. (2019) examinaron la relación entre el gobierno corporativo y el desempeño financiero de instituciones de micro-finanzas (IMF) en Asia. Emplearon un conjunto de datos de panel con 173 entidades, abarcando 18 países asiáticos para el período 2007-2011. Construyeron un índice de gobierno corporativo basado en siete medidas relacionadas con el tamaño y la composición del directorio, las características del CEO y el tipo de propiedad. Posteriormente, estimaron la relación bidireccional entre este índice y cada uno de cinco indicadores de desempeño financiero diferentes. De esa forma, identificaron que la rentabilidad y la sostenibilidad de las IMF tiende a mejorar con las prácticas de buen gobierno. Adicionalmente, encontraron que las IMF más rentables y sostenibles tienen mejores sistemas de gobierno.

Por otra parte, Mertzanis et al. (2019) examinaron el impacto de las instituciones sociales, el gobierno corporativo específico de la empresa y las características de propiedad en el desempeño de la empresa en los países del Medio Oriente y el norte de África (Región MENA). En el análisis utilizaron un conjunto de datos financieros y de gobierno de 225 empresas que cotizan en las bolsas de valores de 11 países de la región MENA para el período 2007-2017. Luego, aplicaron modelos de regresión para probar las hipótesis de investigación. Este estudio sugiere que la relación entre el gobierno corporativo y el desempeño de la empresa depende de la medición utilizada. Sus resultados también mostraron que algunas características específicas de la empresa, como el tamaño de la junta directiva y la propiedad interna e institucional, los niveles de apalancamiento y liquidez son indicadores sólidos del desempeño de la empresa. Sin embargo, si se considera el impacto en la contabilidad y otras medidas de desempeño basadas en el mercado, los resultados no son concluyentes.

Otro conjunto de estudios relacionados con el gobierno corporativo y la teoría de la empresa se han enfocado en aspectos diferentes al desempeño económico. Chang et al. (2014) se enfocaron específicamente en estudiar los efectos de la calidad del gobierno corporativo en la velocidad de ajuste hacia una estructura de capital óptima. Este estudio consideró paralelamente dos efectos de la deuda que se derivan de la teoría de la agencia: la defensa de la adquisición y los efectos disciplinarios de la deuda en la velocidad de ajuste a la estructura de capital óptima. La investigación se enfocó en empresas estadounidenses y abarcó el período 1993-2009. La muestra final incluyó 4297 observaciones por año y se observó cada firma durante al menos dos años consecutivos. Los resultados muestran que el gobierno corporativo tiene un efecto relevante en la velocidad de ajuste de la estructura de capital. En consecuencia, las empresas con gobernanza débil y sobre-endeudadas se ajustan más lentamente hacia el apalancamiento objetivo, en comparación con sus contrapartes de gobernanza fuertes. Esto implica que, las empresas con gobierno débil enfrentan más amenazas de adquisición, tienen menos probabilidades de disminuir el apalancamiento financiero y la deuda total. Así pues, el gobierno corporativo parece ser una herramienta efectiva para mitigar el problema de agencia en el uso de la deuda.

En esta misma línea, Bhagat et al. (2011) profundizaron en el estudio de los efectos de las características del administrador en la estructura del capital de la empresa. Los autores desarrollaron un modelo microeconómico estructural que incluyó empresas globales con datos disponibles en Compustat, ExecuComp, Centro de Investigación de Responsabilidad del Inversor (IRRC) y Centro de Investigación en Precios de Seguridad (CRSP), durante el período 1993-2007. Evaluaron el papel 
de los contratos óptimos del administrador a través de valores financieros que conducen a una estructura de capital dinámica, que refleja los efectos de los impuestos, los costos de bancarrota y los conflictos de la agencia entre el administrador y los accionistas. Identificaron que la deuda a largo plazo disminuye con la capacidad del administrador, la participación accionaria interna y el riesgo a largo plazo de la empresa, pero aumenta con su nivel de riesgo a corto plazo. Además, la deuda a corto plazo disminuye con la capacidad del gerente, aumenta con su participación en el capital y disminuye con el riesgo a corto plazo. En esta misma línea asociada a la estructura de capital, Bae et al. (2011) investigaron la relación que ésta guarda con un índice corporativo de tratamiento a los empleados. La muestra estuvo integrada por empresas que cotizan en bolsa en Estados Unidos entre 1991 y 2007, cubiertas por la Base de datos Sócrates KLD. Encontraron que las compañías que tratan a sus empleados de manera justa mantienen bajos índices de apalancamiento. Este resultado fue robusto a una variedad de especificaciones de modelos y problemas de endogeneidad.

Evidencia para países del sur de Asia ha sido recolectada por Buvanendra et al. (2017). Los autores exploraron los determinantes más importantes de la velocidad de ajuste hacia la estructura de capital óptima de las empresas que cotizan en Sri Lanka e India para el período 2003 a 2013. Un total de 90 empresas que cotizan en la Bolsa de Valores de Colombo (CSE) hicieron parte de la muestra y fueron elegidas en función de la coherencia y disponibilidad de datos. Las empresas de los sectores de banca, finanzas, seguros y servicios públicos fueron eliminadas debido a su naturaleza financiera y comercial. Se empleó un análisis de datos panel para estudiar los factores que influyen en la velocidad de ajuste. Fueron escogidos cinco factores específicos de la empresa (rentabilidad, tamaño, crecimiento, tangibilidad y escudo fiscal) y cinco variables de gobierno corporativo (tamaño de la junta directiva, dualidad del CEO, compensación, propiedad familiar, porcentaje de ejecutivos no directores). Los autores encontraron que la velocidad de ajuste de las relaciones de apalancamiento varía con el tamaño de la brecha de apalancamiento de la empresa, su flujo de caja operativo, su mecanismo de gobierno, su acceso a los mercados de capital y algunos elementos relacionados con las condiciones del mercado. También identificaron que los comportamientos de ajuste de las empresas dependen del país, ya que las empresas de Sri Lanka experimentaron un ajuste más rápido de la estructura de capital que las empresas indias.

Los anteriores trabajos muestran evidencia a nivel internacional de los beneficios económicos y financieros asociados a un buen ejercicio de la gobernanza corporativa. Estos resultados van en línea con nuestro objetivo asociado a medir la existencia de estos beneficios para el caso de las empresas colombianas. Estos beneficios tienen un sustento teórico que recae en la teoría de agencia y la dependencia de recursos, que son corrientes dominantes en el ámbito del gobierno corporativo. A nivel de Latinoamérica y Colombia la evidencia recolectada es relevante. Da Silva y Leal (2005) investigaron la relación entre la calidad de las prácticas de gobierno corporativo de una empresa con su valoración y desempeño, a partir de la construcción de un índice de gobierno corporativo para cada empresa que cotizan en la Bolsa de Valores de Sao Paulo (Bovespa). La muestra final del estudio estuvo integrada por 131 empresas, con información para los años 1998 a 2002. Emplearon análisis de datos de panel y permitieron flexibilidad en la modelación de las diferencias en el comportamiento de las empresas a lo largo del tiempo. Los resultados indicaron que menos del $4 \%$ de las empresas brasileñas presentaban buenas prácticas de gobierno corporativo, y que las compañías con mejor gobierno corporativo evidenciaban un desempeño financiero significativamente mayor. Análogamente, identificaron la existencia de una relación positiva entre la Q de Tobin y las mejores prácticas de gobierno corporativo, no obstante, los resultados no fueron estadísticamente significativos.

A nivel de Colombia, Cortés (2013) examinó la aplicación de las prácticas de gobierno corporativo, definidas en el código de mejores prácticas corporativas (Código País), en los emisores de la Bolsa de Valores de Colombia (BVC) durante el periodo 2007-2011. El objetivo de este trabajo fue determinar si existía alguna relación entre la aplicación de dichas prácticas y el monto demandado de títulos valores por los participantes del mercado. Para tal fin, seleccionó 49 emisores de valores que realizaron 116 procesos de emisión de bonos o acciones y procesó las variables a través de dos modelos de regresión. Al respecto, encontró evidencia que soporta la existencia de una relación entre 
la aplicación de prácticas de gobierno corporativo y el monto total demandado por los inversionistas en el mercado. Esta relación es significativa y ha evolucionado positivamente, principalmente, en los emisores no financieros. Igualmente, identificó que las prácticas de gobierno corporativo relacionadas con la junta directiva ejercen mayor influencia en la demanda de activos financieros; sin embargo, son aplicadas en menor medida por los emisores nacionales.

Con relación a indicadores de desempeño de las empresas colombianas, Benavides y Mongrut (2010) estudiaron los efectos sobre el desempeño contable y las decisiones de financiación de las empresas colombianas después de formular un código de gobierno corporativo. Para ello, estructuraron una base de datos con información de 43 emisores colombianos para el periodo 1997 a 2006. Posteriormente, a partir de un análisis de panel de datos no balanceado evaluaron las hipótesis de mejor desempeño y mayor apalancamiento después de emitir un código. Al respecto, encontraron que el rendimiento de los activos de las empresas después de la introducción del código mejora en más del 1\%. Además, identificaron que el apalancamiento de las empresas aumentó en más del 5\%. Dichos resultados sugieren que la introducción de un código de gobierno corporativo es una medida efectiva para fomentar el desempeño de las empresas en los mercados financieros.

A partir de los resultados de los anteriores trabajos, y en consonancia con la literatura financiera existente, se pueden establecer tres predicciones teóricas. La dirección de las relaciones que se establecen en las predicciones se formula con base en lo que se espera de la teoría de la agencia y la dependencia de recursos. Por ello, se definieron las siguientes hipótesis de investigación:

1. Una mayor calidad de la gobernanza corporativa implica un mejor desempeño financiero de la empresa.

2. Una mayor calidad de la gobernanza corporativa está asociada a un mayor valor de mercadode la empresa.

3. Una mayor calidad de la gobernanza corporativa está asociada con menores niveles de endeudamiento (total o financiero) de la empresa.

En la siguiente sección se muestra la forma en la que, empíricamente, se contrastan cada una de estas hipótesis. En la Sección 4 se presentan los resultados encontrados.

\section{Metodología.}

El presente estudio es un caso de aplicación de la teoría de las finanzas corporativas al estudio de la relación entre el gobierno corporativo, el desempeño de la empresa, su valoración y apalancamiento financiero para firmas que operan en el mercado colombiano. Este se desarrolla específicamente para el caso de compañías colombianas que cotizan en Bolsa y hacen parte del índice bursátil COLCAP. COLCAP es el índice bursátil de referencia de la Bolsa de Valores de Colombia desde enero de 2008 y está compuesto por las 20 acciones más comercializadas en el mercado (Meneses \& Pérez, 2020).

Los datos del estudio se obtuvieron de la plataforma financiera Bloomberg, que recoge datos ambientales, sociales y de gobernanza de compañías que cotizan en bolsa a nivel mundial y, específicamente, para todas las empresas listadas en la Bolsa de Valores de Colombia. La información respectiva de las empresas corresponde a datos anuales entre 2008 y 2018, con fecha de corte a 31 de diciembre.

Para Durst et al. (2019) el análisis y medición del desempeño organizacional es esencial para la supervivencia y el éxito organizacional y, en consecuencia, se espera que todo tipo de organizaciones evalúen las acciones tomadas por los gerentes o directivos. Bajo esa línea de análisis, medir el desempeño proporciona a las organizaciones la retroalimentación necesaria respecto tanto a la eficiencia como a la efectividad de sus actividades, operaciones y esfuerzos y, por lo tanto, pueden 
en el futuro llevar a cabo decisiones más informadas y con menor riesgo. Ahora, está claro que, dependiendo de la organización, el desempeño organizacional puede abarcar elementos tales como servicio al cliente, la administración de costos, el nivel de calidad, la productividad, el desempeño de la administración de activos y el valor de mercado, entre otros (Navimipour et al., 2018). De conformidad con Powell (1992) se asocia el desempeño a la rentabilidad (ROE y ROA) y a partir de Meneses y Macuacé (2011) se incorpora también, el apalancamiento financiero.

Para medir la calidad de la gobernanza se usó un índice de gobierno corporativo diseñado y calculado por Bloomberg, siguiendo la metodología de Da Silva y Leal (2005). Este se denomina el Índice de Gobierno Corporativo (GDS). De acuerdo con Huber et al. (2017) la métrica utilizada por Bloomberg para calcular el desempeño de gobernanza se consolida en el índice GDS; éste toma en cuenta 7 dimensiones de análisis (Remuneración, Independencia, Auditoría, Derechos de los accionistas, Diversidad de la junta directiva, Atrincheramiento y Overboarding) para cada empresa en todas las industrias por medio de una encuesta patentada de datos corporativos, permitiendo realizar un análisis objetivo frente al comportamiento interno de las diversas partes involucradas.

\subsection{Definición de las Variables.}

La Tabla 1 presenta la definición de las variables utilizadas en este documento. Como variables dependientes tenemos: la rentabilidad de los activos (ROA), la rentabilidad del patrimonio (ROE), el valor de la empresa medido por la $\mathrm{Q}$ de Tobin y el nivel de apalancamiento de las empresas (total y financiero). La variable independiente de interés es la calidad en la gobernabilidad de las empresas, aproximado mediante el índice GDS. Adicionalmente, se incluyen algunos controles: las ventas totales (en logaritmos), el margen neto (en porcentaje), la tangibilidad de los activos (en porcentaje) y la deuda financiera de las firmas (en porcentaje).

Tabla 1. Definición de las variables.

\begin{tabular}{|c|c|}
\hline Variables & \\
\hline Descripción & Desempeño \\
\hline ROA & Utilidad neta sobre los activos \\
\hline ROE & Utilidad neta sobre el patrimonio \\
\hline \multicolumn{2}{|l|}{ Valoración } \\
\hline Q de Tobin & Capitalización bursátil más pasivos financieros, sobre el patrimonio \\
\hline \multicolumn{2}{|l|}{ Endeudamiento } \\
\hline Endeudamiento financiero & Pasivos financieros sobre la suma del patrimonio y los pasivos financieros \\
\hline Endeudamiento total & Pasivo total sobre activo total \\
\hline \multicolumn{2}{|l|}{ Gobierno Corporativo } \\
\hline GDS & Índice de gobierno corporativo \\
\hline \multicolumn{2}{|l|}{ Controles } \\
\hline Tamaño & Logaritmo de las ventas totales (millones de COP\$) \\
\hline Tangibilidad & Activos tangibles sobre los activos totales \\
\hline Margen sobre ventas & Utilidad neta sobre ventas totales \\
\hline
\end{tabular}

Fuente: Elaboración propia.

\subsection{Modelo econométrico.}

La primera hipótesis a contrastar en este documento consiste en que una mejor calidad de la gobernanza corporativa en las empresas implica un mayor desempeño. Para esto, siguiendo a Da Silva 
y Leal (2005) y Benavides y Mongrut (2010), se utiliza la especificación econométrica presentada en

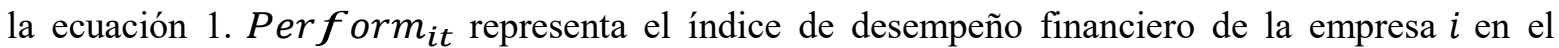
momento $t$, aproximado mediante ROA o ROE, $\boldsymbol{I}_{i t}$ representa el índice de gobernanza para una empresa $i$ en el momento $t$, aproximado mediante GDS, $\boldsymbol{x}_{i t}$ es un vector de controles (tamaño, tangibilidad y margen neto a nivel de empresa), $u_{i t}$ un término de perturbación aleatoria. $\alpha_{0}, \beta$ y $\gamma$ son parámetros para estimar. La significancia estadística y magnitud del parámetro $\beta$ revelará el impacto de $I$ sobre el desempeño de la empresa. Además de los controles mencionados, tal como se hace en Benavides y Mongrut (2010), se deberían tener en cuenta los diversos choques agregados que pueden afectar de manera más o menos homogénea a las empresas, tales como el nivel de actividad económica del país (PIB), la inflación, tasa de cambio, etc. Para esto, en la ecuación 2, se incluyen efectos fijos de tiempo $\left(v_{t}\right)$, con lo cual, se estaría controlando por cualquier factor que varíe a través del tiempo, pero no varíe entre empresas.

$$
\begin{gathered}
\text { Perform }_{i t}=\alpha_{0}+\beta \boldsymbol{I}_{i t}+\boldsymbol{x}_{i t}^{\prime} \boldsymbol{\gamma}+u_{i t} \\
P \text { erform } \\
\text { ert }=\alpha_{0}+\beta \boldsymbol{I}_{i t}+\boldsymbol{x}_{\boldsymbol{i} t}^{\prime} \boldsymbol{\gamma}+v_{t}+u_{i t}
\end{gathered}
$$

En este mismo sentido, es probable que existan factores no observables a nivel de empresa que sean relevantes en la ecuación a estimar. Para controlar por estos, se especifica otro modelo que incluye, además de efectos fijos de tiempo, efectos fijos de empresa $\left(\epsilon_{i}\right)$ :

$$
P \text { erform }_{i t}=\alpha_{0}+\beta \boldsymbol{I}_{i t}+\boldsymbol{x}_{i t}^{\prime} \boldsymbol{\gamma}+v_{t}+\epsilon_{\boldsymbol{i}}+u_{i t} \text {. }
$$

Por otra parte, en este documento también se contrasta si una mayor calidad de la gobernanza corporativa está asociada a un mayor valor de mercado de las empresas, aproximando esta relación mediante la Q de Tobin. Para esto, se especifican dos modelos similares a los anteriores:

$$
\begin{gathered}
Q_{i t}=\alpha_{0}+\beta \boldsymbol{I}_{i t}+\boldsymbol{x}_{i t}^{\prime} \boldsymbol{\gamma}+v_{t}+u_{i t} \\
Q_{i t}=\alpha_{0}+\beta \boldsymbol{I}_{i t}+\boldsymbol{x}_{i t}^{\prime} \boldsymbol{\gamma}+v_{t}+\epsilon_{i}+u_{i t},
\end{gathered}
$$

donde $Q_{i t}$ representa la $\mathrm{Q}$ de Tobin a nivel de empresa.

Finalmente, la tercera hipótesis plantea que la gobernabilidad también afecta el nivel de endeudamiento de las empresas (total o el financiero). Esta hipótesis se contrasta de manera análoga a como se contrastan las dos primeras:

$$
\begin{aligned}
& \text { Leverage }_{i t}=\alpha_{0}+\beta \boldsymbol{I}_{i t}+\boldsymbol{x}_{\boldsymbol{i t}}^{\prime} \boldsymbol{\gamma}+v_{t}+u_{i t}, \\
& \text { Leverage }_{i t}=\alpha_{0}+\beta \boldsymbol{I}_{i t}+\boldsymbol{x}_{i t}^{\prime} \boldsymbol{\gamma}+v_{t}+\epsilon_{\boldsymbol{i}}+u_{i t},
\end{aligned}
$$

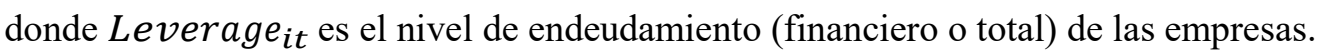

\section{Resultados.}

La Tabla 2 presenta algunas descriptivas de las variables presentadas en la Tabla 1. En ella se presenta el número de observaciones disponibles por variable, la media, desviación estándar y valores extremos. Se destaca que, en promedio, las empresas exhiben un desempeño positivo. Sin embargo, existe una alta variabilidad en el desempeño, independiente de cómo se mida, por la ROA o el ROE. Por otro lado, con relación a la Q de Tobin se destaca que, en promedio, las empresas mantienen una capitalización por debajo de su patrimonio. Sin embargo, debido a la alta variabilidad de esta medida se puede encontrar una gran proporción de empresas que tienen una capitalización por encima de su patrimonio, como se manifiesta por lo alto del valor máximo de esta variable que corresponde a más del doble de capitalización en relación al patrimonio. En cuanto a los niveles de endeudamiento se destaca que, en promedio, las empresas mantienen niveles de endeudamiento relativamente altos, 
cercanos al $60 \%$ en el caso del endeudamiento total. Igualmente, existe una alta variabilidad de las medidas de endeudamiento entre las empresas.

La Figura 1 presenta las correlaciones entre las variables de interés y el indicador de gobierno corporativo. Como se puede observar, el índice de gobierno corporativo (GDS) se correlaciona de forma positiva con los indicadores ROA, ROE, con la Q de Tobin, el margen y la tangibilidad, aunque esta última es cercana a cero; mientras que se correlaciona de forma negativa con el endeudamiento total, el endeudamiento financiero y las ventas. De estas correlaciones, ninguna es fuerte, pues en general no superan el $40 \%$. De igual forma, se muestran las correlaciones entre las otras variables de interés. Destacamos una correlación de $60 \%$ entre la tangibilidad y las medidas de apalancamiento, es decir, cuando se mantienen más activos tangibles las empresas tienen a endeudarse más, lo que es razonable. Además, existen correlaciones negativas de entre $60 \%$ y $80 \%$ por parte de la $\mathrm{Q}$ de Tobin y las medidas de endeudamiento, lo que es razonable.

Tabla 2. Estadísticas Descriptivas.

\begin{tabular}{lccccc}
\multicolumn{1}{c}{ Variable } & Obs. & Media & D.E. & Mín & Máx \\
ROA & 170 & 2.035 & 5.669 & -38.921 & 20.385 \\
ROE & 169 & 7.511 & 11.301 & -70.325 & 33.123 \\
Q de Tobin & 151 & 0.826 & 0.500 & 0.238 & 2.936 \\
Leverage Total & 180 & 58.999 & 21.621 & 3.594 & 93.519 \\
Leverage Fin. & 174 & 41.701 & 19.959 & 0.000 & 84.393 \\
GDS & 152 & 47.709 & 11.013 & 8.929 & 76.786 \\
Ventas & 168 & 28.228 & 3.417 & 18.703 & 31.931 \\
Tangibilidad & 151 & 90.494 & 8.717 & 35.242 & 100.000 \\
Margen & 166 & 9.077 & 15.153 & -74.177 & 57.342 \\
\hline
\end{tabular}

Fuente: Elaboración propia.

Figura 1. Correlaciones entre las variables objeto de estudio.

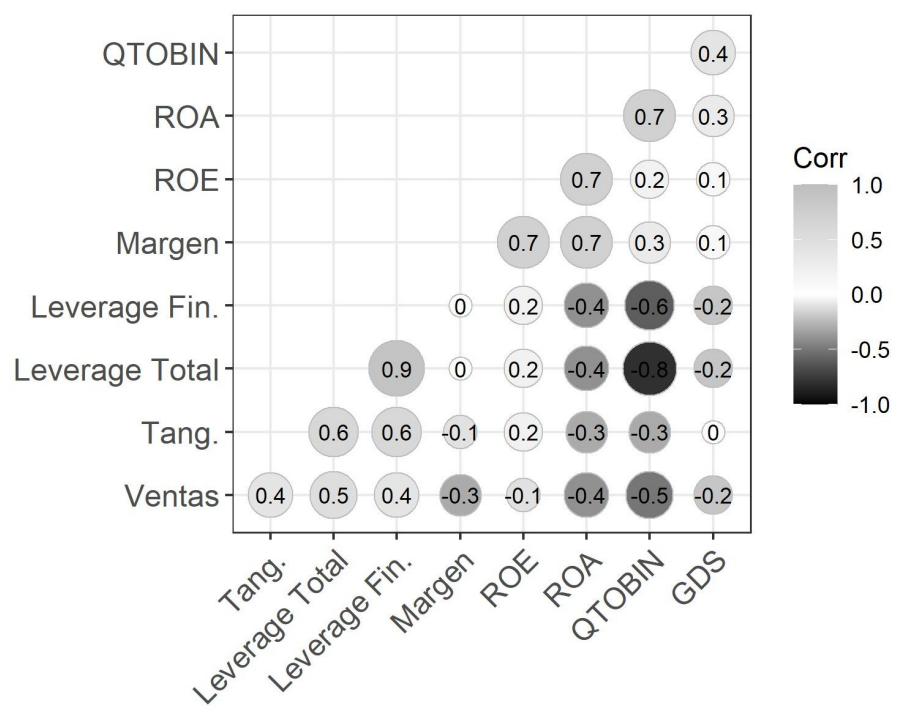

Fuente: Elaboración propia.

Los resultados de los modelos econométricos se presentan en las Tablas 3-5. En la Tabla 3 se muestran los resultados de la estimación de los modelos que relacionan el desempeño de las empresas, aproximado con los indicadores ROA y ROE, frente al índice de gobernanza GDS. Las columnas 1-3 tienen como variable dependiente ROA, y las columnas 4-6 tienen como variable dependiente ROE. 
Para cada columna se menciona cuál de los tres tipos de modelos se ajusta: Pooled, Efectos Aleatorios (RE) y Efectos Fijos (FE). Los resultados muestran que existe una relación positiva entre el índice de desempeño ROA y el indicador de gobernanza GDS. Dicha relación es significativa estadísticamente para los tres tipos de modelos estimados. En cambio, no se encuentra una relación significativa entre el indicador de desempeño ROE y el indicador de gobernanza. Por otro lado, los indicadores de desempeño tienen una relación positiva y significativa con el margen sobre las ventas, y esta relación es robusta al tipo de modelos utilizados.

La relación entre el desempeño de las empresas, medido por el ROE, y la tangibilidad es positiva y significativa, y es robusta al tipo de modelo utilizado. En cuanto a la relación entre desempeño de las empresas y las ventas totales, no se encuentra una relación estadísticamente significativa. Estos resultados muestran que el desempeño de las empresas es más alto cuando éstas tienen mayores márgenes sobre las ventas, una mayor proporción de activos tangibles, y una mejor gobernanza.

Tabla 3. Desempeño de las empresas.

\begin{tabular}{lcccccc}
\hline & $(1)$ & $(2)$ & $(3)$ & $(4)$ & $(5)$ & $(6)$ \\
& ROA & ROA & ROA & ROE & ROE & ROE \\
\hline Ventas & -0.069 & -0.172 & 1.278 & 0.002 & -0.064 & $5.672^{*}$ \\
& $(0.117)$ & $(0.170)$ & $(1.258)$ & $(0.318)$ & $(0.231)$ & $(3.117)$ \\
Tangibilidad & $-0.117^{* * *}$ & 0.014 & $0.066^{* *}$ & $0.179^{* * *}$ & $0.174^{* * *}$ & $0.230^{* *}$ \\
& $(0.024)$ & $(0.029)$ & $(0.030)$ & $(0.055)$ & $(0.054)$ & $(0.091)$ \\
Margen & $0.203^{* * *}$ & $0.267^{* * *}$ & $0.268^{* * *}$ & $0.486^{* * *}$ & $0.555^{* * *}$ & $0.547^{* * *}$ \\
& $(0.026)$ & $(0.043)$ & $(0.043)$ & $(0.058)$ & $(0.093)$ & $(0.099)$ \\
GDS & $0.110^{* * *}$ & $0.058^{* * *}$ & $0.048^{*}$ & -0.015 & -0.010 & 0.024 \\
& $(0.030)$ & $(0.021)$ & $(0.028)$ & $(0.061)$ & $(0.052)$ & $(0.065)$ \\
Constante & $7.946^{*}$ & -0.908 & -44.974 & -12.352 & -11.776 & $-175.211^{* *}$ \\
& $(4.648)$ & $(4.647)$ & $(34.690)$ & $(11.715)$ & $(7.921)$ & $(85.314)$ \\
\hline Obs & 121.000 & 121.000 & 121.000 & 121.000 & 121.000 & 121.000 \\
R2 ajustado & 0.675 & & 0.902 & 0.611 & & 0.779 \\
Modelo & Pooled & $\mathrm{RE}$ & $\mathrm{FE}$ & Pooled & $\mathrm{RE}$ & $\mathrm{FE}$ \\
\hline
\end{tabular}

Nota: Errores estándar robustos entre paréntesis.

$* * * \mathrm{p}<0.01, * * \mathrm{p}<0.05, * \mathrm{p}<0.1$

Fuente: Elaboración propia.

En la Tabla 4, se presentan las regresiones estimadas que relacionan la Q de Tobin a nivel de empresa con el índice GDS, para los tres tipos de modelos: Pooled, RE y FE. Los resultados muestran que el índice de gobernanza tiene una relación positiva y significativa con la $\mathrm{Q}$ de Tobin. Dicha relación es robusta al tipo de modelo utilizado. Por otro lado, existe una relación no significativa entre la Q de Tobin con los márgenes sobre las ventas; no es clara con la tangibilidad y las ventas. Estos resultados muestran que el valor de las empresas es mayor cuando éstas tienen una buena gobernanza, lo que es razonable dada la teoría que se ha planteado en este trabajo. Con las otras variables no se encuentra una relación clara. 
Tabla 4. Q de Tobin.

\begin{tabular}{|c|c|c|c|}
\hline & $\begin{array}{c}(1) \\
Q \text { de Tobin }\end{array}$ & $\begin{array}{c}(2) \\
Q \text { de Tobin }\end{array}$ & $\begin{array}{c}(3) \\
Q \text { de Tobin }\end{array}$ \\
\hline Ventas & $\begin{array}{l}-0.101 * \\
(0.057)\end{array}$ & $\begin{array}{c}-0.202 * * \\
(0.096)\end{array}$ & $\begin{array}{l}-0.270 \\
(0.177)\end{array}$ \\
\hline Tangibilidad & $\begin{array}{c}-0.019 * * * \\
(0.006)\end{array}$ & $\begin{array}{c}0.003 \\
(0.009)\end{array}$ & $\begin{array}{c}0.015 * * * \\
(0.005)\end{array}$ \\
\hline Margen & $\begin{array}{l}-0.001 \\
(0.003)\end{array}$ & $\begin{array}{c}0.002 \\
(0.005)\end{array}$ & $\begin{array}{c}0.006 \\
(0.005)\end{array}$ \\
\hline GDS & $\begin{array}{c}0.023 * * * \\
(0.004)\end{array}$ & $\begin{array}{c}0.015 * * * \\
(0.005)\end{array}$ & $\begin{array}{l}0.011 * \\
(0.006)\end{array}$ \\
\hline Constante & $\begin{array}{c}4.388 * * * \\
(1.603)\end{array}$ & $\begin{array}{c}6.129 * * \\
(2.740)\end{array}$ & $\begin{array}{c}7.125 \\
(5.250)\end{array}$ \\
\hline $\begin{array}{l}\text { Obs } \\
\text { R2 ajustado } \\
\text { Modelo }\end{array}$ & $\begin{array}{c}107.000 \\
0.542 \\
\text { Pooled }\end{array}$ & 107.000 & $\begin{array}{c}107.000 \\
0.862 \\
\mathrm{FE}\end{array}$ \\
\hline
\end{tabular}

Finalmente, en la Tabla 5, se presentan las regresiones para el nivel de apalancamiento de las empresas, total y financiero. Los resultados muestran que el apalancamiento, total y financiero, tiene una relación negativa y significativa con el indicador de gobernanza GDS. Por otra parte, no existe una relación clara entre los niveles de apalancamiento, total y financiero, con las ventas y los márgenes sobre éstas. Para la tangibilidad, la relación con el apalancamiento total es positiva, pero solo es significativa para los modelos Pooled y RE. Estos resultados muestran que el nivel de apalancamiento de las empresas es menor cuando éstas mantienen buenas prácticas de gobernanza.

Tabla 5. Apalancamiento total (T) y financiero (F).

\begin{tabular}{lcccccc}
\hline & $(1)$ & $(2)$ & $(3)$ & $(4)$ & $(5)$ & $(6)$ \\
& $\begin{array}{c}\text { Leverage } \\
\text { Total }\end{array}$ & $\begin{array}{c}\text { Leverage } \\
\text { Total }\end{array}$ & $\begin{array}{c}\text { Leverage } \\
\text { Total }\end{array}$ & $\begin{array}{c}\text { Leverage } \\
\text { Fin. }\end{array}$ & $\begin{array}{c}\text { Leverage } \\
\text { Fin. }\end{array}$ & $\begin{array}{c}\text { Leverage } \\
\text { Fin. }\end{array}$ \\
\hline Ventas & -0.114 & 1.576 & $13.423^{* * *}$ & -0.911 & 0.533 & 4.096 \\
& $(0.544)$ & $(1.024)$ & $(4.363)$ & $(0.620)$ & $(1.078)$ & $(5.293)$ \\
\multirow{2}{*}{ Tangibilidad } & $1.711^{* * *}$ & $0.482^{* * *}$ & 0.172 & $1.584^{* * *}$ & $0.365^{* *}$ & -0.128 \\
& $(0.217)$ & $(0.150)$ & $(0.121)$ & $(0.200)$ & $(0.150)$ & $(0.146)$ \\
Margen & $0.334^{* * *}$ & -0.037 & $-0.271^{* * *}$ & $0.317^{* * *}$ & -0.085 & $-0.318^{* * *}$ \\
& $(0.102)$ & $(0.090)$ & $(0.092)$ & $(0.098)$ & $(0.097)$ & $(0.111)$ \\
GDS & $-0.798^{* * *}$ & $-0.343^{* * *}$ & $-0.202^{*}$ & $-0.748^{* * *}$ & $-0.285^{* * *}$ & -0.134 \\
& $(0.110)$ & $(0.095)$ & $(0.111)$ & $(0.124)$ & $(0.104)$ & $(0.116)$ \\
& $-54.805^{* *}$ & -26.442 & $-325.391 * * *$ & -40.553 & -11.858 & -66.756
\end{tabular}




\begin{tabular}{lcccccc}
\hline Obs & 121.000 & 121.000 & 121.000 & 121.000 & 121.000 & 121.000 \\
R2 ajustado & 0.539 & & 0.925 & 0.503 & & 0.897 \\
Modelo & Pooled & RE & FE & Pooled & RE & FE \\
\hline
\end{tabular}

Nota: Errores estándar robustos entre paréntesis. $* * * \mathrm{p}<0.01, * * \mathrm{p}<0.05, * \mathrm{p}<0.1$

Fuente: Elaboración propia.

\subsection{Especificación de los modelos.}

Dado que se tienen tres tipos de modelos (Pooled, RE, FE), en esta sección se establece cuál de los tres modelos es el preferido, por lo que se determinaría cuáles serían los principales hallazgos encontrados en las Tablas 3-5. En la Tabla 6 establecemos, bajo el criterio de elección de la Prueba de Especificación de Hausman, la elección del modelo preferido entre los tres utilizados para las 5 variables dependientes de interés. Destacamos que el modelo preferido para las medidas de desempeño es RE, y para Q de Tobin y las medidas de endeudamiento es FE. Esto tiene las siguientes consecuencias:

- Existe una relación positiva entre el desempeño de las empresas y la gobernanza corporativa. Además, las medidas de desempeño se relacionan de forma positiva con la tangibilidad y el margen de ventas.

- El valor de la empresa tiene una relación positiva con la gobernanza corporativa y la tangibilidad.

- El endeudamiento tiene una relación negativa con la gobernanza corporativa y los márgenes sobre las ventas. Adicionalmente, se encuentra una relación positiva entre endeudamiento y las ventas.

Tabla 6. Prueba de Especificación de Hausman.

\begin{tabular}{lc}
\hline V. dependiente & Modelo preferido \\
\hline ROA & RE \\
ROE & RE \\
Q de Tobin & FE \\
Endeudamiento total & FE \\
Endeudamiento financiero & FE \\
\hline
\end{tabular}

Fuente: Elaboración propia.

Finalmente, en las Tablas 7 y 8 se muestran las pruebas de autocorrelación y heterocedasticidad para panel de datos. Las hipótesis nulas de las pruebas son no autocorrelación y homocedasticidad. Por la Tabla 7 encontramos evidencia de que no se rechaza la hipótesis nula de no autocorrelación, sin embargo, por la Tabla 8 encontramos evidencia de que se rechaza la hipótesis de homocedasticidad, es decir, nuestros modelos estimados tienen heterocedasticidad. Por ello, nosotros corregimos los errores estándar de las Tablas 3-5 para que estos sean robustos a la heterocedasticidad. 
Tabla 7. Prueba de Autocorrelación.

\begin{tabular}{cccccc}
\hline Rezagos & ROE & ROA & Q de Tobin & E. total & E. financiero \\
\hline 1 & 0.038 & 0.127 & 0.022 & 0.099 & 0.044 \\
2 & 0.26 & 0.341 & 0.352 & 0.022 & 0.415 \\
3 & 0.121 & 0.101 & 0.161 & 0.409 & 0.826 \\
4 & 0.975 & 0.145 & 0.806 & 0.06 & 0.049 \\
5 & 0.148 & 0.147 & 0.83 & 0.073 & 0.064 \\
\hline
\end{tabular}

Fuente: Elaboración propia.

Tabla 8. Prueba de Heterocedasticidad.

\begin{tabular}{lr}
\hline V. Dependiente & p-valor \\
\hline ROE & 0.0000 \\
ROA & 0.0000 \\
Q de Tobin & 0.0000 \\
E. total & 0.0000 \\
E. financiero & 0.0000 \\
\hline
\end{tabular}

Fuente: Elaboración propia.

\section{Conclusiones y discusión.}

Este artículo constituye una contribución a la literatura financiera que documenta la relación entre el gobierno corporativo y el desempeño de la empresa en el marco de los mercados emergentes. Se analizó una muestra de empresas colombianas que cotizan en bolsa y hacen parte del índice bursátil COLCAP. La calidad de la gobernanza se midió a partir de un índice de propiedad de Bloomberg que integra variables que representan mecanismos internos y externos asociados al gobierno corporativo. Adicionalmente, se implementaron técnicas de análisis econométrico para panel de datos no balanceados. Se incluyeron efectos fijos de empresa y año para controlar por posibles factores inobservables que afecten la relación entre desempeño de las empresas y el indicador de gobernanza corporativa.

Los resultados obtenidos permiten evidenciar que las diferentes actividades estratégicas asociadas a una mayor calidad del gobierno corporativo son determinantes relevantes de un buen desempeño organizacional. El índice de gobernabilidad (GDS), empleado en este estudio, se correlaciona de manera positiva con la rentabilidad de las empresas. Este hallazgo se suma a la amplia lista de investigaciones en el campo de las finanzas que han corroborado esta relación positiva, tanto a nivel de mercados desarrollados como emergentes (Escobar et al., 2016; Benavides \& Mongrut, 2010; Brown \& Caylor, 2004; Iqbal et al., 2019).

De igual manera, existe una relación significativa entre la calidad del gobierno corporativo, medida por el índice GDS, frente al valor de la empresa, representado por la Q de Tobin. Dicha evidencia ha sido corroborada por la literatura financiera contemporánea a nivel de los mercados desarrollados, que sostiene que la maximización del valor de la empresa no obedece exclusivamente a políticas netamente financieras. Valores adicionales como el fortalecimiento de actividades directivas que generan confianza y transparencia en el mercado son relevantes para mejorar el desempeño de las empresas. Este resultado es acorde a lo encontrado por Brown y Caylor (2004) en el mercado estadounidense y Da Silva y Leal (2005) en el mercado brasilero, pero opuesto a los hallazgos de Benavides y Mongrut (2010) para el mercado colombiano. 
Nuestra investigación encontró que las empresas con mejor calificación en el índice de gobernabilidad mantienen bajos niveles de endeudamiento. Esta relación negativa entre apalancamiento y gobierno corporativo permite apreciar que las empresas que utilizan criterios GDS generan retornos más sostenibles en el largo plazo convirtiéndose en más atractivas para los inversionistas, por lo que hacen menos uso del endeudamiento financiero. Otra lectura posible, de acuerdo con Bae et al. (2011), se asocia al factor reputacional. Las empresas que tienen altos incentivos para mantener su reputación en los mercados financieros tienden a mantener menores niveles de apalancamiento financiero. Por otra parte, se encontró que el margen neto ejerce un efecto negativo y significativo sobre el nivel de endeudamiento. Esto implica que el apalancamiento se reduce con la rentabilidad de las empresas. Este hallazgo reviste alta importancia para los investigadores de la estructura de capital y es consistente con las investigaciones de Frank y Goyal (2009), Fama y French (2002) y Benavides et al. (2016).

En la presente investigación, sólo se incluyeron dentro de las empresas objeto de estudio, firmas o corporaciones que cotizan en el mercado de valores colombiano y hacen parte del índice bursátil COLCAP. Otro tipo de firmas se excluyen debido a las dificultades en la consecución de información sobre las prácticas de gobierno corporativo dado que no hacen parte de los índices de gobernanza estimados por Bloomberg. Para investigaciones futuras, se recomienda consolidar estudios para analizar las prácticas de gobierno corporativo y su incidencia en el desempeño empresarial a nivel de Latinoamérica, tomando como referencia al Mercado Integrado Latinoamericano MILA. Además, se sugiere evaluar la consistencia de los resultados, comparando la calificación GDS suministrada por dos o más plataformas de información financiera.

\section{Referencias}

Acero, I., \& Alcalde, N. (2012). Gobierno corporativo y rendición de cuentas: ¿existe algún efecto sobre la performance empresarial? Revista de Contabilidad-Spanish Accounting Review, 15(1), 143178.

Bae, K., Kang, J., \& Wang, J. (2011). Employee treatment and firm leverage: A test of the sta- keholder theory of capital structure. Journal of Financial Economics, 100(1), 130-153.

Benavides, J., Berggrun, L., \& Perafan, H. (2016). Dividend payout policies: evidence from latin america. Finance Research Letters, 17, 197-210.

Benavides, J. \& Mongrut, S.M. (2010). Governance codes: facts or fictions? A study of governance codes in Colombia. Estudios Gerenciales, 26(117), 85-102.

Bhagat, S., Bolton, B., \& Subramanian, A. (2011). Manager characteristics and capital structure: Theory and evidence. Journal of Financial and Quantitative Analysis, 46(6), 1581-1627.

Brown, L.D. \& Caylor, M.L. (2004). Corporate governance and firm performance. Georgia State University. Working Paper, 1-53. http://dx.doi.org/10.2139/ssrn.586423

Buvanendra, S., Sridharan, P., \& Thiyagarajan, S. (2017). Firm characteristics, corporate governance and capital structure adjustments: A comparative study of listed firms in Sri-Lanka and India. IIMB management review, 29(4), 245-258.

Castro, R.M., \& Cano, M.A. (2004). Buen gobierno corporativo, solución a la crisis de confianza: Cambios en la contabilidad y la auditoría, aporte de los estándares internacionales, apuntes de la comparación de los casos parmalatenron. Contaduría universidad de Antioquia, 44, 17-51. 
Chang, Y., Chou, R.K., \& Huang, T. (2014). Corporate governance and the dynamics of capital structure: New evidence. Journal of Banking and Finance, 48, 374-385.

Cortés, D.L. (2013). Análisis de las prácticas de Gobierno Corporativo en la Bolsa de Valores de Colombia. AD-minister, 23, 25-43.

Da Silva, A.C.L. \& Leal, R.P.C. (2005). Corporate governance index, firm valuation and performance in Brazil. Brazilian Review of Finance, 3(1), 1-18.

Durst, S., Hinteregger, C., \& Zieba, M. (2019). The linkage between knowledge risk management and organizational performance. Journal of Business Research, 105, 1-10.

Escobar, N., Benavides, J., \& Perafán, H. (2016). Corporate governance and financial performance: theoretical concepts and empirical evidence. Cuadernos de Contabilidad, 17(43), 203-254.

Fama, E.F. \& French, K.R. (2002). Testing trade-off and pecking order predictions about dividends and debt. The review of financial studies, 15(1), 1-33.

Frank, M.Z. \& Goyal, V.K. (2009). Capital structure decisions: which factors are reliably important? Financial management, 38(1), 1-37.

Haynes, K. \& Hillman, A. (2010). The effect of board capital and CEO power on strategic change. Strategic Management Journal, 31, 1145-1 163.

Holmstrom, B. (1979). Moral hazard and observability. Bell journal of Economics, 10(1), 74-91.

Huber, B.M., Comstock, M., \& Polk, D. (2017). ESG Reports and Ratings: What They Are, Why They Matter. The Harvard Law School Forum on Corporate Governance. https://corpgov.law.harvard.edu/2017/07/27/esg-reports-and-ratings-what-they-are-why-theymatter/

Iqbal, S., Nawaz, A., \& Ehsan, S. (2019). Financial performance and corporate governance in microfinance: Evidence from Asia. Journal of Asian Economics, 60, 1-13.

Jensen, M.C., \& Meckling, W.H. (1976). Theory of the firm: Managerial behavior, agency costs and ownership structure. Journal of Financial Economics, 3(4), 305-360.

Meneses, L., \& Macuacé, R. (2011). Valoración y riesgo crediticio en Colombia. Revista Finanzas y Política Económica, 3(2), 65-82.

Meneses, L., \& Pérez, C. (2020). Análisis comparativo de eficiencia en mercados emergentes. el casode Colombia, Chile y Perú. Apuntes Contables, 26(26), 9-24. https://doi.org/10.18601/16577175.n26.02

Mertzanis, C., Basuony, M.A., \& Mohamed, E.K. (2019). Social institutions, corporate governanceand firm-performance in the MENA region. Research in International Business and Finance, 48, 7596.

Méndez, J.A., \& Rivera, H.A. (2015). Relación entre gobierno corporativo y posicionamiento organizacional: instituciones de educación superior en américa latina. Educación y educadores, $18(3), 435-455$.

Navimipour, N.J., Milani, F.S., \& Hossenzadeh, M. (2018). A model for examining the role of effective factors on the performance of organizations. Technology in Society, 55, 166-174. 
OCDE (2004). Principios de Gobierno Corporativo. París: OECD Publishing. https://doi.org/10.1787/9789264259171-es

Oneto, A., \& Núñez, G. (2015). Corporate Governance in Brazil, Chile, Colombia, Mexico and Peru. The Determinants of Risk in Corporate Debt Issuance. Economic Commission for Latin America and the Caribbean (ECLAC). https://scioteca.caf.com/handle/123456789/698

Paz, C. (2004). El gobierno corporativo como estrategia de creación de valor. Revista de Derecho Mercantil, 251, 7-65.

Pfeffer, J., \& Salancik, G.R. (2003). The External Control of Organizations: A Resource Dependence Perspective. Stanford University Press. https://www.gsb.stanford.edu/facultyresearch/books/external-control-organizations-resource-dependence-perspective

Powell, T.C. (1992). Research notes and communications strategic planning as competitive advantage. Strategic Management Journal, 13(7), 551-558. 